\title{
Perfiles y signos de radicalización de las mujeres radicalizadas por organizaciones terroristas de etiología yihadista en España.
}

Profiles and signs of radicalization of women radicalized by terrorist organizations of jihadist etiology in Spain

\author{
Ariadna Trespaderne ${ }^{1}$ \\ David Garriga ${ }^{1}$
}

(1) Comunidad de Inteligencia y Seguridad Global; Barcelona, España.

Email correspondencia: info@intelciseg.com

\section{Resumen}

En los últimos años, la investigación académica ha ido ampliando su objeto de estudio en relación a los perfiles vinculados a la radicalización violenta. Ahora, la mujer se percibe como un actor activo en la radicalización violenta, la captación y las actividades terroristas. El presente estudio describe el perfil de las 21 mujeres investigadas por radicalización de etiología yihadista en España, sus signos de radicalización y las características presentes según las funciones ejercidas dentro de la organización terrorista. Los reslutados obtenidos indican que existe una diferencia en el perfil de las mujeres según su origen, sus signos externos de radicalización y funciones dentro de la organización terrrosita.

Palabras Clave: radicalización violenta. terrorismo de etiologia yibadista. perfil femenino. signos de radicalización. funciones.

\begin{abstract}
In recent years, the academy has been expanding its object of study in relation to profiles linked to violent radicalization. Now, women are perceived as active actors in violent radicalization, recruitment and terrorist activities. The present study describes the profile of the 21 women investigated for radicalization of jihadist etiology in Spain, its signs of radicalization and the present characteristics according to the functions exercised within the terrorist organization. The obtained results indicate that there is a difference in the profile of the women according to their origin, their external signs of radicalization and functions within the terrrosite organization.
\end{abstract}

Keywords: violent radicalization. terrorism of jihadist etiology. female profile. signs of radicalization. functions 


\section{INTRODUCCIÓN}

$\mathrm{Si}$ bien las organizaciones terroristas han estado formadas mayoritariamente por miembros del sexo masculino, a mitades del siglo pasado, se inicia un cambio de tendencia y el rol femenino empieza a potencializarse (Osborne, 2017). Al Qaeda, mantuvo el papel de la mujer fuera de la yihad bélica y su función era acompañar al hombre en su camino de lucha y sacrificarse por él. Debían ser diligentes y focalizarse en labores de apoyo hacia la organización terrorista más que en la lucha activa y combatiente. De este modo, educaban a los niños, seguían la senda de Dios, enfatizaban su amor por la yihad, ayudaban económicamente a aquellos combatientes presos y también, a las familias que tuvieran hombres desplazados.

Hoy por hoy, la militancia de mujeres en organizaciones terroristas de etiología yihadista ya no es una excepción. Su incorporación, se vincula al conflicto emergente en Siria e Irak y al Daesh. Estas mujeres comparten los objetivos y medios de la organización terrorista, y asumen un papel activo para promover el mal llamado Califato (GarcíaCalvo, 2017). El Daesh ha ensalzado de un modo alarmante la yihad femenina y su participación activa: (i) por querer construir un pseudo estado, donde es imprescindible la esposa y la madre, y (ii) frente la necesidad de nuevos combatientes activos a raíz de la represión bélica y la presión militar sufrida (Jacoby, 2015). Así, la presencia de la mujer aparece como un papel estratégico y con una dimensión simbólica. A mediados de 2015, su participación activa se dispara y en 2016, se confirman los primeros casos de mujeres combatientes del Daesh. Esto se debe a la ampliación de su rol doméstico y su concepción. El uso de mujeres combatientes sirve al Daesh para desmarcarse de otras organizaciones terroristas de etología yihadista, enfatizar la determinación de sus miembros y distinguirse por su estrategia criminal (Eggert, 2015).

Pese a la importancia de tal problemática, existen pocas fuentes bibliográficas que tratan específicamente la contribución de la mujer en el campo de batalla y en las organizaciones terroristas. Sin embargo, gracias a la evolución y cambio de actitudes de las mujeres en la comunidad musulmana, la cuestión es algo mucho más actual y visible. Si bien se hallan estudios sobre las manifestaciones más espectaculares, como son las mujeres suicidas (Speckhard, 2008; Bloom, 2011; Campana, 2014), cierto es que las formas menos singulares del radicalismo violento en mujeres son poco estudiadas e inadecuadamente documentadas. Se establece la necesidad de abordar nuevas líneas estratégicas para la prevención (Jordán, 2009; Rafiq y Malik, 2015).
Según Pearson y Winterbotham (2017) la radicalización violenta difiere entre mujeres y hombres. Por ello, el Centre for the Prevention of Radicalization Leading to Violence (2016) advierte de la necesidad de conocer todas las formas de radicalización femenina, sus funciones y posiciones que ocupan dentro de la organización terrorista. Todo ello, desde una perspectiva de género para así poder prevenir y combatir más adecuadamente cada una de sus formas ya sea en infantes, hombres o mujeres. Si bien no existe un perfil psicológico terrorista (Nesser, 2006; Kruglanski y Fishman, 2009) y tampoco un único perfil homogéneo de mujeres pertenecientes al Daesh (Saltman y Smith, 2015), existen características comunes en la mayoría de casos del Estado Español.

Según García-Calvo (2017), durante las detenciones en España entre los años 2013 y 2016 relacionadas con el terrorismo de etiología yihadista, un $16,9 \%$ son mujeres con una edad media de 24 años, siete puntos por debajo de los hombres. El 73,3\% de éstas, comprendían la edad de 19 a 28 años, siendo éste el rango de edad más destacado. De un modo más desglosado, el 47,9\% de éstas tenían de 19 a 23 años y un $26,2 \%$, entre 24 y 28 años. Otros datos de interés, es que entre los 14 a 18 años hay un 13\% de mujeres donde existieron varias detenciones y en una de ellas, era menor de 14 años. También constan detenciones a mujeres que superan los 44 años -8,6\%-, la más adulta de 52 años. Solamente un 4,3\% tenían entre 29 y 33 años. Tales datos, no difieren a las experiencias de otros países occidentales. Esto es así, porque la edad media en el inicio del proceso de radicalización es a los 20,7 años. En términos generales, las nacionalidades más destacadas entre ellas son la española y la marroquí. Un dato destacado es el estado civil ya que el $45 \%$ de estas mujeres estaban solteras -un 16,6\% más que los hombres-, un $25 \%$ casadas -al contrario de los hombres, que el $61,4 \%$ están casados-, un 10\% separadas o en unión de hecho y otro $10 \%$ viudas.

La misma autora señala, por un lado, que el 60,9\% de estas mujeres son de nacionalidad española y el 56,5\% de éstas, son nacidas en España. El 65,2\% de ellas eran residentes del territorio nacional y de segundas generaciones de Melilla y Ceuta. Otro 34,8\% son de nacionalidad marroquí y el 39,1\% de éste, nacidas en Marruecos. Por otro lado, el 13\% eran mujeres conversas, sin ascendencia familiar, cultural o religiosa de esa naturaleza. Además, el $87,5 \%$ de las mujeres poseían estudios secundarios y un 6,3\% de estudios superiores. En el momento de su detención, el 26,5\% de éstas estaban cursando estudios. El 33,3\% estaban en paro y las que trabajaban estaban generalmente en el sector de servicios. 
A diferencia de los hombres, ninguna de estas mujeres tenía antecedentes penales. Las detenciones e investigaciones de estas mujeres se ubican en la zona de ponente, siendo Cataluña, Valencia y Andalucía focos de interés. Otros puntos geográficos que deben ser relevantes son, Ceuta y Melilla. Todo lo anterior guarda una relación directa con la estrategia de las organizaciones terroristas en captar y radicalizar a mujeres jóvenes de edades fértiles para establecerse en los territorios ocupados y que contraigan matrimonio y construyan su familia allí. Además, responde a cuestiones de vulnerabilidad, ya que son mujeres en una etapa donde aún están gestando su identidad y se encuentran en un proceso de formación.

El presente estudio tiene por objeto a) describir el perfil de las mujeres investigadas por radicalización de etiología yihadista en España, b) clasificar las diferentes investigadas según su función dentro del grupo de etiología yihadista y c) analizar los signos de radicalización emergentes entre los diferentes perfiles de mujeres radicalizadas.

\section{MÉTODO}

\section{A. Participantes}

La muestra está formada por 21 mujeres, el total de investigadas por radicalización de grupos de etiología yihadista en España en los últimos diez años. Los criterios de inclusión fueron: a) ser investigada por radicalización violenta en España con o sin sentencia condenatoria y b) poseer funciones de reclutadora o bien, de ser captada para convertirse en una combatiente extranjera.

\section{B. Procedimiento}

La información necesaria se obtuvo de distintas fuentes abiertas OSINT, informes periciales y entrevistas semiestructuradas con su núcleo familiar y red social de apoyo. Los datos recogidos hacen referencia a factores biopsico-sociales de dichas mujeres, entre ellos: edad, estado civil, procedencia, población, provincia y comunidad autonómica donde reside, nivel de estudios, situación laboral, entorno familiar, cambios conductuales y físicos durante la radicalización, entre otros.

\section{Instrumento}

Para la recodificación de las variables y el posterior análisis de datos, se empleó el programa estadístico IBM SPSS Statistics 20 para realizar un análisis descriptivocorrelacional.

\section{RESLUTADOS}

Los resultados obtenidos indican que la mayor parte de las mujeres tienen entre los 19 y 25 años $(28,6 \%)$, seguido de menores de edad (14,3\%) y mayores de 25 años $(9,5 \%)$. Sin embargo, casi la mitad de la muestra $(47,6 \%)$ no consta tal información debido a la dificultad de obtención de datos OSINT.

Tabla 1. Edades de las mujeres investigadas por terrorismo de etiología yihadista en España.

\begin{tabular}{ccc}
\hline \hline & Frecuencia & Porcentaje \\
\hline \hline Meores de 18 & 3 & 14,3 \\
\hline De 19 a 25 & 6 & 28,6 \\
\hline Mayores de 25 & 2 & 9,5 \\
\hline Desconocido & 10 & 47,6 \\
\hline Total & 21 & 100,0 \\
\hline
\end{tabular}

De igual modo, se observa un mayor porcentaje de mujeres solteras $(57,1 \%)$ por encima de las casadas $(38,1 \%)$.

Tabla 2. Estado civil de las muje.res investigadas por terrorismo de etiología yihadista en España

\begin{tabular}{ccc}
\hline & Frecuencia & Porcentaje \\
\hline \hline Casada & 8 & 38,1 \\
\hline Soltera & 12 & 57,1 \\
\hline Desconocido & 1 & 4,8 \\
\hline Total & 21 & 100,0 \\
\hline
\end{tabular}

Se identifica una presencia casi nula en las primeras generaciones de musulmanes $(4,8 \%)$. La mayoría de ellas son segundas generaciones $(66,7 \%)$ y destaca la presencia de un porcentaje significativo de mujeres autóctonas del país $(28,6 \%)$.

Tabla 3. Tipo de generación de las mujeres investigadas por terrorismo de etiología yihadista en España.

\begin{tabular}{ccc}
\hline & Frecuencia & Porcentaje \\
\hline \hline Primera & 1 & 4,8 \\
\hline Segunda & 14 & 55,7 \\
\hline Autóctona & 6 & 28,6 \\
\hline Total & 21 & 100,0 \\
\hline
\end{tabular}

Se observa que la mayoría de mujeres no tienen hijos $(81 \%)$, frente a un $20 \%$. De entre ellas, el $14,3 \%$ tienen un hijo y el $4,8 \%$ dos. 
Tabla 4. Número de hijos de las mujeres investigadas por terrorismo de etiología yihadista en España.

\begin{tabular}{ccc}
\hline \hline & Frecuencia & Porcentaje \\
\hline \hline Uno & 3 & 14,3 \\
\hline Dos & 1 & 4,8 \\
\hline Ninguo & 17 & 81,0 \\
\hline Total & 21 & 100,0 \\
\hline
\end{tabular}

Casi la mitad de las mujeres $(47,6 \%)$ poseen funciones de reclutamiento hacia otras mujeres para prepararlas a viajar a zonas de conflicto. La otra mitad mujeres radicalizadas y convencidas son para unirse al grupo terrorista.

Tabla 5. Función dentro de la organización terrorista de las mujeres investigadas por terrorismo de etiología yihadista en España.

\begin{tabular}{ccc}
\hline \hline & Frecuencia & Porcentaje \\
\hline \hline Reclutamiento & 10 & 47,6 \\
\hline Combatiente & 11 & 52,4 \\
\hline Total & 21 & 100,0 \\
\hline
\end{tabular}

Es importante analizar si el tipo de vestimenta se relaciona con el nivel de radicalización violenta, ya que la mujer la cual opta por la prenda del Niqab -prenda que cubre íntegramente a la mujer y solo deja a la vista los ojosposee un mayor grado de escalamiento dentro de la radicalización en comparación a las mujeres que emplean el Hiyab -clásico pañuelo en la cabeza que solo cubrir cabello y hombros-. Se comprueba que, en el caso español, hay una mayor presencia de Niqabs (85,7\%) que Hiyabs (14,3\%).

Tabla 6. Tipología de velo de mujeres investigadas por terrorismo de etiología yihadista en España.

\begin{tabular}{ccc}
\hline & Frecuencia & Porcentaje \\
\hline \hline Hiyab & 18 & 85,7 \\
\hline Niqab & 3 & 14,3 \\
\hline Total & 21 & 100,0 \\
\hline
\end{tabular}

La presencia del Niqab en las mujeres radicalizadas es presente en poblaciones como Ceuta y Melilla, pero también en poblaciones catalanas como Barcelona. El uso del Hiyab sí que está presente en todas las poblaciones por igual. Según el origen de la mujer investigada, se valora que el Niqab está presente en las mujeres de origen marroquí, siendo nulo en las de origen español.
Tabla 7. Relación entre la vestimenta y el origen de las mujeres investigadas por terrorismo de etiología yihadista en España.

\begin{tabular}{|c|c|c|c|c|c|}
\hline & & & \multicolumn{2}{|c|}{ Vestimienta } & \multirow[t]{2}{*}{ Total } \\
\hline & & & Hiyab & Niqab & \\
\hline \multirow{12}{*}{ Origen } & \multirow[t]{4}{*}{ Marroquí } & Recuento & 12 & 3 & 15 \\
\hline & & $\begin{array}{l}\% \text { dentro de } \\
\text { Origen }\end{array}$ & 80 & 20 & 100,0 \\
\hline & & $\begin{array}{l}\% \text { dentro de } \\
\text { Vestimienta }\end{array}$ & 66,7 & 100,0 & 71,45 \\
\hline & & $\%$ del total & 57,16 & 14,29 & 71,45 \\
\hline & \multirow[t]{4}{*}{ Española } & Recuento & 6 & 0 & 6 \\
\hline & & $\begin{array}{c}\% \text { dentro de } \\
\text { Origen }\end{array}$ & 100,0 & 0 & 100,0 \\
\hline & & $\begin{array}{l}\% \text { dentro de } \\
\text { Vestimienta }\end{array}$ & 33,3 & 0 & 28,6 \\
\hline & & $\%$ del total & 28,6 & 0 & 28,6 \\
\hline & \multirow[t]{4}{*}{ Total } & Recuento & 18 & 3 & 21 \\
\hline & & $\begin{array}{l}\% \text { dentro de } \\
\text { Origen }\end{array}$ & 85,7 & 14,3 & 100,0 \\
\hline & & $\begin{array}{l}\% \text { dentro de } \\
\text { Vestimienta }\end{array}$ & 100,0 & 100,0 & 100,0 \\
\hline & & $\%$ del total & 85,7 & 14,3 & 100,0 \\
\hline
\end{tabular}

Para valorar si existen signos de radicalización en las mujeres, se han analizado los datos sobre cambios físicos y conductuales del sujeto que accede a un programa de radicalización por parte de una organización terrorista como Al-Qaeda o Daesh (Garriga, 2015). Se observa que dos terceras partes de la muestra $(66,7 \%)$, presentan claramente diversos signos de radicalización.

Tabla 8. Presencia de signos radicalización en las mujeres investigadas por terrorismo de etiología yihadista en España.

\begin{tabular}{ccc} 
& Frecuencia & Porcentaje \\
\hline \hline Presencia de cambios & 14 & 66,7 \\
\hline Ausencia de cambios & 7 & 33,3 \\
\hline Total & 21 & 100,0 \\
\hline
\end{tabular}

Existen diferencias en el origen -marroquí o español-, entre aquellas mujeres radicalizadas que pretenden incorporarse y vincularse a una organización terrorista para ser mujeres de muyahidines, en comparación con aquellas mujeres que desean trabajar como reclutadoras desde Occidente. Una cuarta parte de las investigadas en España son de origen español y claramente apreciamos la mínima presencia de conversas en los cargos de reclutamiento, la mayoría de ellas son de origen marroquí. En cambio, en las candidatas a incorporarse a la organización terrorista se observa que se equilibra el origen, siendo prácticamente igual el porcentaje. 
Tabla 9. Relación entre la función y el origen de las mujeres investigadas por terrorismo de etiología yihadista en España,

\begin{tabular}{|c|c|c|c|c|c|}
\hline & & & \multicolumn{2}{|c|}{ Origen } & \multirow[t]{2}{*}{ Total } \\
\hline & & & Marroquí & $\begin{array}{c}\text { Españo } \\
1\end{array}$ & \\
\hline \multirow{8}{*}{ Función } & \multirow[t]{4}{*}{ Reclutamiento } & Recuento & 9 & 1 & 10 \\
\hline & & $\begin{array}{l}\% \text { dentro de } \\
\text { Función }\end{array}$ & 90,0 & 10,0 & 100,0 \\
\hline & & $\begin{array}{c}\% \text { dentro de } \\
\text { Origen }\end{array}$ & 60,0 & 16,7 & 47,6 \\
\hline & & $\%$ del total & 42,9 & 16,7 & 47,6 \\
\hline & \multirow[t]{4}{*}{ Incorporación } & Recuento & 6 & 5 & 11 \\
\hline & & $\begin{array}{l}\% \text { dentro de } \\
\text { Función }\end{array}$ & 54,5 & 45,5 & 100,0 \\
\hline & & $\begin{array}{l}\% \text { dentro de } \\
\text { Origen }\end{array}$ & 40,0 & 83,3 & 52,4 \\
\hline & & $\%$ del total & 28,6 & 23,8 & 52,4 \\
\hline \multirow{4}{*}{\multicolumn{2}{|c|}{ Total }} & Recuento & 15 & 6 & 21 \\
\hline & & $\begin{array}{l}\% \text { dentro de } \\
\text { Función }\end{array}$ & 71,4 & 28,6 & 100,0 \\
\hline & & $\begin{array}{c}\% \text { dentro de } \\
\text { Origen }\end{array}$ & 100,0 & 100,0 & 100,0 \\
\hline & & $\%$ del total & 71,4 & 28,6 & 100,0 \\
\hline
\end{tabular}

No existen diferencias significativas entre las funciones de aquellas mujeres que poseen uno o varios hijos. Observamos así, que el número de hijos está presente de igual forma tanto en las mujeres candidatas a la incorporación como las elegidas para reclutar. Sin embargo, en muchos de los perfiles la utilización de mujeres reclutadoras con hijos pueden acceder más fácilmente a otras mujeres en edades fértiles y con hijos pequeños. Aunque solo el 20\% tienen hijos, únicamente un $40 \%$ de la muestra está casada, por lo que estaríamos frente a un $50 \%$ de mujeres casadas con infantes.

Tabla 10. Relación entre la función y el número de hijos de las mujeres investigadas por terrorismo de etiología yihadista en España.

\begin{tabular}{|c|c|c|c|c|c|c|}
\hline & & & \multicolumn{2}{|c|}{ Hijos } & & \multirow[t]{2}{*}{ Total } \\
\hline & & & Uno & Dos & No & \\
\hline \multirow{8}{*}{ Función } & \multirow[t]{4}{*}{ Reclutamiento } & Recuento & 1 & 1 & 8 & 10 \\
\hline & & $\begin{array}{l}\% \text { dentro de } \\
\text { Función }\end{array}$ & 10,0 & 10,0 & 80,0 & 100,0 \\
\hline & & $\begin{array}{c}\% \text { dentro de } \\
\text { Hijos }\end{array}$ & 33,3 & 100,0 & 47,1 & 47,6 \\
\hline & & $\%$ del total & 4,8 & 4,8 & 38,1 & 47,6 \\
\hline & \multirow[t]{4}{*}{ Incorporación } & Recuento & 2 & 0 & 9 & 11 \\
\hline & & $\begin{array}{l}\% \text { dentro de } \\
\text { Función }\end{array}$ & 18,2 & 0 & 81,8 & 100,0 \\
\hline & & $\begin{array}{c}\% \text { dentro de } \\
\text { Hijos }\end{array}$ & 66,7 & 0 & 52,9 & 52,4 \\
\hline & & $\%$ del total & 28,6 & 23,8 & & 52,4 \\
\hline \multirow{4}{*}{\multicolumn{2}{|c|}{ Total }} & Recuento & 3 & 1 & 17 & 21 \\
\hline & & $\begin{array}{l}\% \text { dentro de } \\
\text { Función }\end{array}$ & 14,3 & 4,8 & 81,0 & 100,0 \\
\hline & & $\begin{array}{c}\% \text { dentro de } \\
\text { Hijos }\end{array}$ & 100,0 & 100,0 & 100,0 & 100,0 \\
\hline & & $\%$ del total & 14,3 & 4,8 & 81,0 & 100,0 \\
\hline
\end{tabular}

Se valora que una mujer presenta signos de radicalización violenta, cuando muestra al menos tres de los cambios físicos o conductuales establecidos por Garriga (2015). Se observa una diferencia significativa en aquellas mujeres reclutadoras, que no muestran ningún signo de radicalización $(60 \%)$, frente a aquellas mujeres que son captadas para incorporarse a la organización terrorista que si presentan signos de radicalización (90,9\%). Esto se debe a que las mujeres reclutadoras están aconsejadas para el uso de la Takbia o el engaño, para pasar desapercibidas en occidente. Así, se visten de un modo discreto y que no resalte su radicalización.

Tabla 11. Relación entre la función y los signos de radicalización de las mujeres investigadas por terrorismo de etiología yihadista en España

\begin{tabular}{|c|c|c|c|c|c|}
\hline & & & \multicolumn{2}{|c|}{ Radicalización } & \multirow[t]{2}{*}{ Total } \\
\hline & & & $\mathrm{Si}$ & No & \\
\hline \multirow{8}{*}{ Función } & \multirow[t]{4}{*}{ Reclutamiento } & Recuento & 4 & 6 & 10 \\
\hline & & $\begin{array}{l}\% \text { dentro de } \\
\text { Función }\end{array}$ & 40,0 & 60,0 & 100,0 \\
\hline & & $\%$ dentro de Origen & 28,6 & 85,7 & 47,6 \\
\hline & & $\%$ del total & 19,0 & 28,6 & 47,6 \\
\hline & \multirow[t]{4}{*}{ Incorporación } & Recuento & 10 & 1 & 11 \\
\hline & & $\begin{array}{l}\% \text { dentro de } \\
\text { Función }\end{array}$ & 71,4 & 14,3 & 52,4 \\
\hline & & $\%$ dentro de Origen & 71,4 & 14,3 & 52,4 \\
\hline & & $\%$ del total & 48,6 & 4,8 & 52,4 \\
\hline \multirow{4}{*}{\multicolumn{2}{|c|}{ Total }} & Recuento & 14 & 7 & 21 \\
\hline & & $\begin{array}{l}\% \text { dentro de } \\
\text { Función }\end{array}$ & 66,7 & 33,3 & 100,0 \\
\hline & & $\%$ dentro de Origen & 100,0 & 100,0 & 100,0 \\
\hline & & $\%$ del total & 66,7 & 33,3 & 100,0 \\
\hline
\end{tabular}

\section{DISCUSIÓN}

Los resultados obtenidos concuerdan con García-Calvo (2017). La mayor parte de las mujeres radicalizadas comprenden el intervalo de edad de los 19 a 25 años, en su mayoría solteras y sin hijos, de segundas y terceras generaciones. Cuando están casadas, tienen como máximo dos hijos. Casi la mitad de las mujeres poseen funciones de reclutamiento, y la otra mitad o bien mostraban una participación activa en la organización terrorista, o estaban siendo preparadas para un futuro desplazamiento a zonas de conflicto. Las mujeres reclutadoras eran en su mayoría de origen marroquí, y las radicalizadas incorporadas, de origen español.

Tras los resultados obtenidos se confirma la existencia de cambios en los rasgos físicos y conductuales de las mujeres investigadas en España por radicalización violenta 
de etiología yihadista durante su proceso de transformación en comparación a los indicadores obtenidos por Garriga (2015). Mediante las entrevistas a familiares y amigos directos de las investigadas, se notifica la presencia de tales cambios claramente visibles para la gente que las conocía con anterioridad. Tal información, nos dota de un recurso importante para poder detectar cuándo un individuo se halla en un proceso de radicalización y a su vez, poder aplicar medidas preventivas para ayudar a detener este proceso de pensamiento polarizado típico en este tipo de fanatización.

En su mayoría, empleaban Niqabs en su vestimenta, indicando una evolución en el uso del velo según su nivel de radicalización: de Hiyab a Niqab, de Niqab a Burka. Además, se podían observar otros cambios de apariencia física como la falta de uso de adornos, joyas y accesorios femeninos, ausencia de tatuajes visibles, retirada del uso de colonias con alcohol y eliminación de cualquier símbolo cristiano. De igual modo, pueden apreciarse otras alteraciones en el comportamiento individual como la intensificación de la práctica religiosa, el estricto horario de rezo, el uso intensificado de retórica religiosa y política, cambios de trabajo, alteraciones en sus relaciones familiares, retraimiento y polarización social, exposición selectiva a medios de comunicación, entre otros. Se añaden también otros comportamientos colectivos como el intercambio o consumo de propaganda, nuevas interacciones con grupos cerrados online y de mensajería instantánea, entre otros. Destacar que la mayor parte de mujeres reclutadoras no poseían signos de radicalización externa, pero sí son apreciables en las mujeres vinculadas a un proceso de radicalización para unirse activamente a la organización terrorista.

También es importante valorar que casi la totalidad de las mujeres, sobre todo las de incorporación a la organización terrorista, presentaban estos cambios tanto a nivel físico como comportamental, apoyando la necesidad, cada vez más evidente, de formación a nivel social. La prevención, a día de hoy radica en la sociedad civil, ya que son los observadores directos y continuos de los cambios de las investigadas. Sin embargo, sin una formación adecuada para saber qué es lo que tienen que ver, es difícil poder detectar y parar a tiempo este proceso.

De igual modo, se confirma que existen diferencias entre los rasgos bio-psico-sociales de las mujeres investigadas en España, las cuáles ejercían como reclutadoras o eran miembros activos en la organización terrorista. Esto nos aporta una herramienta fundamental en el seguimiento y control desde occidente en las mujeres radicalizadas, ya tengan funciones de incorporación activa o de reclutamiento. Primero, el tipo de contra narrativa a emplear para que no se unan a estas estructuras criminales, supuestamente idilicas, mediante la erosión, deslegitimación y desmitificación del discurso terrorista. Y segundo, como presentan características distintas, el modo de detección y seguimiento variará según sean reclutadoras o solo estén siendo reclutadas para la causa terrorista.

Por tanto, es de vital importancia detectar a las mujeres sumergidas en un proceso de radicalización, pero también, aquellas mujeres encargadas de reclutar a otras. Observando las posibilidades en detección que ofrece el estudio, debe incidirse en crear mecanismos de prevención eficaces para evitar futuras radicalizaciones. Para la detección de cambios conductuales y rasgos físicos se incluyen cuarto líneas de actuación. Primero, formar a los actores sociales en la detección de indicadores vinculados con la radicalización y el extremismo violento. Segundo, fomentar el control informal en las zonas comunitarias donde confluyen un mayor número de perfiles vulnerables mediante profesionales del ámbito psico-social. Tercero, potenciar la inclusión social y la inserción socio-laboral de los potenciales perfiles para disminuir el riesgo de radicalización violenta y a su vez, favorecer la desradicalización. Cuarto, establecer nuevas líneas de comunicación con el objetivo de generar confianza entre las comunidades, la sociedad civil y los actores gubernamentales.

De acuerdo con Jordán (2009) deben desarrollarse mecanismos de prevención eficaces como: (i) establecer políticas contra la radicalización y la capitación violenta en entornos claves -lugares de culto, zonas ocio, redes sociales, entorno familiar, entre otros-, (ii) construir una estrategia comunicativa homogénea para la contra narrativa en la Unión Europea, (iii) desarrollar narrativas no emotivas y de carácter cientifico con el fin de discutir el fenómeno y no fomentar la discriminación, demonización y la polarización entre la comunidad musulmana y occidente y (iv) elaborar líneas de investigación europeas en clave de género sobre los factores de riesgo relacionados con la radicalización violenta en mujeres. Coincidiendo con Rafiq y Malik (2015) debe reducirse la difusión de la propaganda terrorista, fomentar la resiliencia hacia ésta y dotar de herramientas preventivas en el entorno cercano del sujeto vulnerable para detener los procesos de radicalización violenta en estadios poco avanzados. Así, se converge con las recomendaciones preventivas propuestas por los autores. Por un lado, políticas proactivas como la concienciación social sobre la manipulación existente en la propaganda terrorista 
femenina así como la necesidad de fortalecer el vínculo de las madres de los potenciales perfiles radicalizados con los actores sociales de la comunidad mediante talleres, grupos de apoyo, formaciones mixtas, entre otros. Por otro lado, las políticas reactivas como fomentar la contra narrativa en los medios de comunicación y a su vez, facilitar la creación de centros sanitarios y sociales para la atención de las mujeres en riesgo de radicalización y aquellas familias que detecten posibles cambios conductuales $\mathrm{y} / \mathrm{o}$ físicos de su familiar.

En relación a las limitaciones del presente estudio, la muestra escogida son aquellas mujeres investigadas por radicalización violenta en España con o sin sentencia condenatoria. La muestra no debe ser extrapolada al perfil de mujeres investigadas en otros países europeos ni tampoco al perfil de aquellas mujeres no investigadas por las instituciones. Para futuras investigaciones, se propone realizar un análisis comparativo para determinar si existen diferencias entre las investigadas por radicalización violenta en función de los distintos países de la Unión Europea.

\section{CONLUSIONES}

Con base a todo lo expuesto con anterioridad, el crecimiento de mujeres dentro de organizaciones terrorista supone una amenaza potencial al incorporarlas en la lucha activa, tanto las mujeres radicalizadas que regresan a países occidentales como las mujeres desplazadas a otras zonas las cuales luchan por la causa (Loken y Zelenz, 2018). Esto aumenta la probabilidad de tomar iniciativas propias y realizar ataques -individuales o grupales- en sociedades europeas, ya sean realizados por miembros de la organización terrorista o bien, lobos solitarios.

Es importante seguir investigando este fenómeno desde una perspectiva de género para estudiar la radicalización violenta femenina. Tal y como manifiesta Ennaji (2016), para contrarrestar las narrativas terroristas debe comprenderse antes los motivos de la radicalización violenta tanto en hombres como en mujeres.

Combatir esta problemática es responsabilidad de múltiples actores gubernamentales, institucionales y de la propia sociedad civil. Se requiere de un enfoque integral con medidas no solo militares, policiales y judiciales sino también, programas que aborden factores de riesgo personales, sociales y culturales (Peresin y Cervone, 2015).

La educación y la contranarrativa deben ser dos enfoques fundamentales para combatirlo, ya que existe el uso de una propaganda y promesas explícitas hacia estas para radicalizarlas. Los estudios indican que los actores sociales clave para ello son las comunidades y las familias, así como las mujeres son potentes agentes de prevención, detección, desradicalización y de resiliencia (Naciones Unidas, 2012; Eggert, 2015; Osborne, 2017; Dietrich y Carter, 2017). Como se ha podido vislumbrar en esta investigación, la captación y radicalización violenta fémina no es una réplica a la masculina, y para ello deben trabajarse también, programas de prevención y desradicalización centrados en sus circunstancias particulares (Saltman y Smith, 2015).

\section{REFERENCIAS}

Bloom, M. (2011). Bombshell: The Many Faces of Women Terrorists. Toronto: Penguin Canada.

Campana, A. (2014). Victimes ou bourreaux? Étude comparée des représentations médiatiques des femmes kamikazes dans onze médias francophones et anglophones de 1985 à 2010. En A. Gingras (ed.), Genre et politique dans la presse en France et au Canada, (pp. 145-168). Quebec: Presses de l'Université du Québec.

Centre for the Prevention of Radicalization Leading to Violence. (2016). Women and Violent Radicalization Research Report. Québec: Conseil du statut de la femme.

Dietrich, L., y Carter, S. E. (2017). Gender and Conflict Analysis in ISIS Affected Communities of Iraq. Reino Unido, UK: Oxfam International.

Eggert, J. P. (2015). Women fighters in the "Islamic State" and al-Qaida in Iraq: a comparative analysis. Journal of International Peace and Organization, 90(3-4), 363-380.

Ennaji, M. (2016). Recruitment of foreign male and female fighters to Jihad: Morocco's multifaceted counter-terror strategy. International Review of Sociology, 26(3), 546-557.

García-Calvo, C. (2017). No hay vida sin yihad y no hay yihad sin hégira": la movilización yihadista de mujeres en España, 2014-2016. ARI n 33/2015, Real Instituto Elcano. Recuperado el 28 de abril de 2018 desde http://www.realinstitutoelcano.org/wps/wcm/connect/2b8022ebac17-4145-abfe-e0b6d33c72ce/ARI28-2017-GarciaCalvomovilizacion-yihadista-mujeres-Espana-20142016.pdf?MOD=AJPERES\&CACHEID=2b8022eb-ac17-4145abfe-e0b6d33c72ce.

Garriga, D. (2015). Yihad ¿Qué es?. Barcelona: Editorial Comanegra.

Jacoby, T. A. (2015). Jihadi brides at the intersections of contemporary feminism. New Political Science, 37(4), 525-542.

Jordán, J. (2009a). Políticas de prevención de la radicalización violenta en Europa: elementos de interés para España. Revista Electrónica de Ciencia Penal y Criminología, (11), 1-25.

Kruglanski, A. W., y Fishman, S. (2009). Psychological factors in terrorism and counterterrorism: Individual, group, and organizational levels of analysis. Social Issues and Policy Review, 3(1), 144.

Loken, M., y Zelenz, A. (2018). Explaining extremism: Western women in Daesh. European Journal of International Security, 3(1), 45-68.

Naciones Unidas. (2012). Wherever There Is Conflict, Women Must Be Part of the Solution, Security Council Told in Day-Long Debate Urging Their Inclusion 

Journal, 4(1), 21-28.

in Restoring Fractured Societies. Recuperado el 22 de abril de 2018 desde https://www.un.org/press/en/2012/sc10840.doc.htm

Nesser, P. (2006). Islamist Terrorism in Europe. London: Hurts and Company

Osborne, A. (2017). Gender Equality and Radicalisation in the West AsiaNorth Africa Region. Jordan: WANA Institute, Royal Scientific Society in Amman.

Pearson, E., y Winterbotham, E. (2017). Women, Gender and Daesh Radicalisation: A Milieu Approach. The RUSI Journal, 162(3), 60-72.

Peresin, A., y Cervone, A. (2015). The western muhajirat of ISIS. Studies in Conflict \& Terrorism, 38(7), 495-509
Rafiq, H., \& Malik, N. (2015). Caliphettes: las mujeres, objeto y sujeto de la llamada de DAESH. London: Quiliam Foundation. Recuperado de http://www.

quilliamfoundation.org/wp/wpcontent/uploads/publications/free /caliphettes-spanish.pdf

Saltman, E. M., y Smith, M. (2015). Till Martyrdom Do US Part? Gender and the ISIS Phenomenon. London: ICSD.

Speckhard, A. (2008). The emergence of female suicide terrorists. Studies in Conflict and Terrorism, 31(11), 995-1023. 